\title{
Code + Chords: Targeting Self-Efficacy in Music Technology (WIP)
}

\section{Alyssa Marie Eggersgluss, Playful Learning Lab}

Alyssa Eggersgluss is a K-12 Vocal Music Education Major from the University of St. Thomas. Passionate about interdisciplinary learning, she works as a part of Dr. AnnMarie Thomas' Playful Learning Lab to create learning opportunities for students. She is currently focused on exploring different ways to engage students with sound.

\section{Dr. AnnMarie Thomas, University of St. Thomas}

AnnMarie Thomas is a professor in the School of Engineering and the Opus Colluege of Business at the University of St. Thomas where she is the director of the UST Center for Engineering Education. Her research group, the Playful Learning Lab, focuses on engineering and design education for learners of all ages.

\section{Dr. Deborah Besser P.E., University of St. Thomas}

Dr. Besser, PE, ENV SP, holds a PhD in education and MS and BS in civil engineering. Currently, she is civil engineering chair and Center for Engineering Education director. Previous experience includes faculty positions in diverse universities where she has taught a variety of coursework including steel, timber, concrete and masonry design, construction, engineering economy, engineering graphics and engineering education. Prior to teaching, Dr. Besser, a licensed engineer, was a design engineer with HNTB-CA, where she worked on seismic retrofits and new design of high profile transportation structures.

\section{Rachel Farah, University of St. Thomas}

I am a computer engineering student at the University of Saint Thomas and am a researcher at the Playful Learning Lab. I am passionate about educating and promoting self efficacy with the youth across all socioeconomic levels.

Mr. Cullen Charles Kittams, The Playful Learning Lab

Ms. Emma Michelle Monson, University of St. Thomas

Emma Monson is an undergraduate studying Elementary Education with a STEM co-major at the University of St. Thomas.

\section{Ms. Krista Schumacher, University of St. Thomas}

Krista is an undergraduate Elementary Education and STEM Education major at the University of St. Thomas.

\section{Dr. Jeff Jalkio, University of St. Thomas}

Jeff Jalkio is currently a physics professor at the University of St. Thomas. Jeff worked for thirteen years in industry in the fields of optical sensor design and process control. In 1984, he co-founded CyberOptics Corporation, where he led engineering efforts as Vice President of Research. In 1997 he returned to academia, joining the engineering faculty of the University of St. Thomas and has taught courses in electronics, digital system design, mathematics, physics, circuit theory, electromagnetics, statistical process control, computing, mechatronics, control theory, metrology and design. 


\title{
Code + Chords: Targeting Self-Efficacy in Music Technology (Work in Progress)
}

\begin{abstract}
Primary school students can benefit from feeling capable and confident that they could succeed in computer engineering. This study used music and creation-based learning in exploration of the computer program Code + Chords to help achieve that goal. Code + Chords is a customizable music visualization software that responds to the pitch and amplitude of a person's voice, giving users the interdisciplinary experience of working with coding, music, and technology. This research project was based on the question, "To what extent may self-efficacy be increased in music technology using Code + Chords programming?" Primary school participants explored music, coding, and technology at three St. Paul Public Libraries. These two-hour-long workshops fostered learning through multiple hands-on activities and use of the program Processing to support engagement with the Code + Chords program. Pre- and post-workshop surveys were taken to analyze student's experiences with the workshop as a whole. Survey results showed that the average participant's self-efficacy minutely decreased. Written observations of positive student engagement with Code + Chords alongside survey results suggest that, with improvements, there is strong potential for increased self-efficacy in future iterations of this workshop.

\section{Background}

This study aims to explore self-efficacy in music technology by having participants engage with a program called Code + Chords. Self-efficacy is a person's confidence that they can succeed at a task or within a subject. Code + Chords is a music visualization software based in the programming language Processing that responds in real time to the pitch and amplitude of a monophonic (single pitch) input, such as one voice. The Code + Chords team from the University of St. Thomas's Playful Learning Lab developed the software as a visual accompaniment to specific songs for Cantus' performance at the Science Museum of Minnesota. After receiving positive feedback from this performance, the team decided that the software would benefit from further development and use.

In the summer of 2018, Code + Chords shifted their focus away from the visual development of the software and toward educational outreach using the software. Educational outreach began through a collaboration with the Playful Learning Lab (PLL), Metro Deaf School (MDS) and Public Broadcasting Service (PBS) to create an episode for the PBS show called SciGirls. In this episode, PLL employees taught three students from MDS how to code a unique visual into the Code + Chords software. Throughout the process of filming, it was clear how confident and proud the girls were growing to be of their work in music technology. After that collaboration, Code + Chords workshops were held for ages 6 through 18 in both formal and informal education settings. Each workshop supported a claim that selfefficacy in music technology might be positively affected through engagement with Code + Chords, but no conclusive data was drawn. In November 2019, the Code + Chords team began this study to gain data evaluating the effectiveness of one variation of a Code + Chords workshop in increasing self-efficacy in music technology.

Purpose 
By giving students the tools to intertwine coding with music, it was hoped that the workshops would cause students to better understand their learning abilities and become more confident in their ability to succeed in technology- and music-related fields. Al Salami [1] explains the need to increase student interest in STEM fields. STEM fields are becoming increasingly prevalent in the job market with a shortage of experienced STEM workers to fill open positions. Al Salami [1] writes that many schools are transitioning to a more integrated curriculum to get students involved at a young age with concepts and ideas within STEM fields. The Code + Chords workshop that this study is focused on uses an integrated curriculum by combining music with technology and takes it one step further by targeting self-efficacy in participants.

Denise Green [2] illustrates the need for understanding and increasing self-efficacy in students:

"Researchers in this area provide empirical evidence that self-efficacy is an accurate predictor of a student's skill acquisition, rate of performance, expenditure of energy, persistence, goal setting, and selfmonitoring of goals. [2]" By designing an integrated curriculum focused on increasing self-efficacy, Green [2] and Al Salami's [1] studies suggest that students positively affected by the workshops could later excel as capable workers within numerous important and available STEM jobs.

\section{Related Works}

Many K-12 schools are pushing to increase technological knowledge to better prepare students for careers in the $21^{\text {st }}$ century, though some schools are still exploring ways to do so. Some of these schools equate technological knowledge with programming knowledge. "Every Child a Coder? Research Challenges for a 5-18 Programming Curriculum" [3] highlights the increase in programming education and the necessity of knowledge of coding in day-to-day life. With coding knowledge becoming more essential, this paper shows the challenges of developing a specific way to teach programming. The Code + Chords workshop suggested in this study offers one approach that would address some of the questions asked in their [3] workshop scope. Most applicably, it would draw on knowledge from other subject areas (music) in order to deepen comprehension in computer science.

Code + Chords hoped to teach technological knowledge through programming using a music visualization software; hence the use of the phrase 'music technology'. By using the arts to support technological learning, researchers hypothesized that not only would academic knowledge increase but self-efficacy in music technology would also increase. Green [2] found statistically significant data in her study exploring the relationship between self-efficacy and sense of membership in relation to arts education in New York high: "The implications from the study indicated that arts education classes and activities were essential to the development of students' critical thinking skills and self-concept growth [2]." The findings from this study show that self-efficacy is increased in arts classes. This study, combined with the findings of previous studies [1], [2] gave us reason to believe that tying coding and music visualization (the arts) together through Code + Chords would increase self-efficacy in the field of music technology.

\section{Methods}

The research question that this Code + Chords study sought to answer was, "To what extent may selfefficacy be increased in music technology using Code + Chords programming?" It was hypothesized that, after completing the workshops, participants would have higher self-efficacy in music technology than when they arrived. To test this question, researchers conducted three two-hour-long workshops at 
various public libraries in St. Paul, Minnesota. These workshops were composed of five sections which each targeted a different topic applicable to music technology with Code + Chords. During these workshops, data was collected through various surveys and written observations about workshop events. The researchers were four undergraduate students from the University of St. Thomas' Playful Learning Lab, with degrees in Civil Engineering, Computer Engineering, Mechanical Engineering, and Music Education. All four had previous experience teaching music technology using Code + Chords software.

The workshops in this study were advertised to children ages 7-17 in order to potentially compare the results from different age groups, but the participants that attended were all within the age range of 7-13. The first workshop had five participants, the second had nine participants, and the third had five participants. At the beginning of the workshop, students were asked to choose a code name of their choosing and briefed about what Code + Chords software is, as well as the agenda of the workshop. Researchers made sure that all participants knew that their participation was voluntary and could be revoked at any time during the workshop. To avoid coercion, participants were reminded of this continually throughout the workshop and while taking the surveys.

An entry survey of four short response questions and four scale questions was given to each participant at the start of the workshop. Scale questions asked participants to circle a number between one and ten that represented their answer, with one meaning "very little" and 10 meaning "a lot". Through this information, researchers gathered insight about the students and their experience with music technology prior to the workshops. The workshop concluded with an exit survey of three short response questions and six scale questions regarding their experience with the workshop. Some questions were duplicates of questions in the entry survey while some questions were new. The purpose of asking repeated questions was to assess change in participant self-efficacy after completing this workshop. In addition to an entry and exit survey, participants were asked to respond to three scale questions in post-activity surveys at five points during the workshop. These surveys aimed to assess the effect of each activity within the workshop on self-efficacy.

\section{Results}

This study had nineteen total participants; a sample size too small to draw any statistical significance from. Because of this, survey results were analyzed using the raw average change of participant responses. This was done by finding the increase, decrease, or lack of change in point values between participants' first and second answers to a question. Answers were given on a scale from one to ten with one being "very little" and ten being "a lot". The changes in self-efficacy for all participants were found, and point values of those changes were averaged to create the raw average change. If a participant did not respond to this question, their score was marked as a 0 , indicating for their result that self-efficacy neither increased nor decreased. After these workshops, there was an average decrease of .0789 points in confidence for all participants. If only the participants that responded to both a pre-workshop and postworkshop survey are considered in the results, this study shows an average decrease of .125 points in confidence for participants.

A self-reflection on increased computer programming knowledge was included on the post-survey response. Of the responses [Figure 1], three said their understanding increased very little, three said their understanding increased a lot, and the 13 remaining participants stated varying degrees of increased understanding within the given range. The average of the group was a 5.3, suggesting that the group as a whole moderately increased their understanding of programming. 


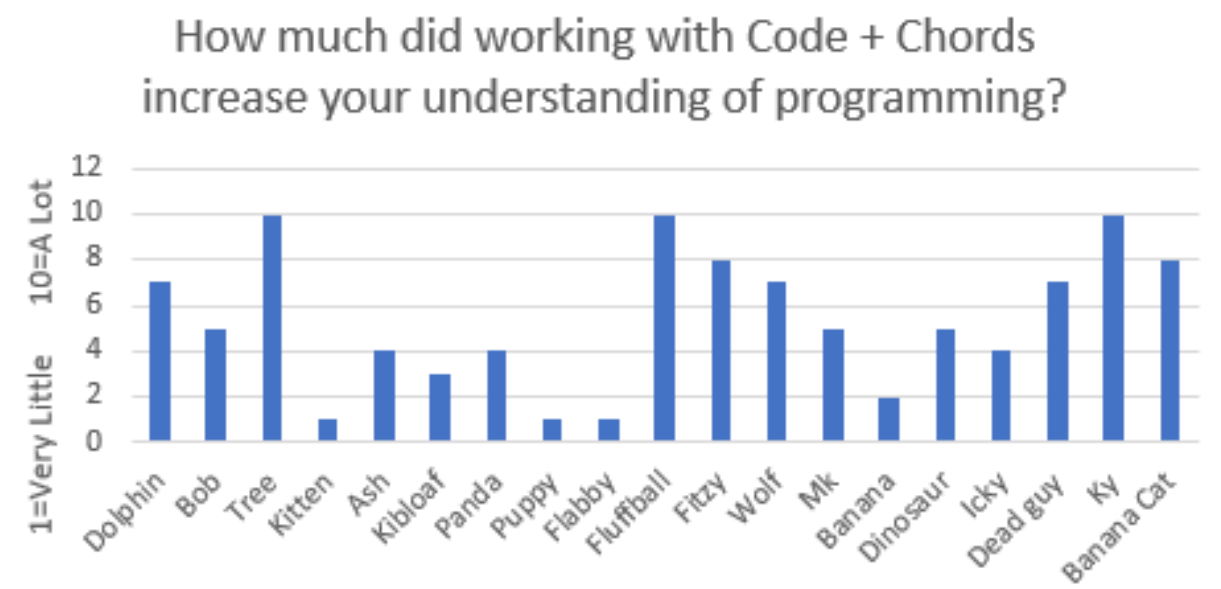

Figure 1: A post-workshop survey response to the question, "How much did working with Code + Chords increase your understanding of programming?" for all participants in the study.

An important question asked in the exit survey was, "Did Code + Chords software allow your experience with music to be more enjoyable?" Of those responses [Figure 2], 10 participants gave a positive affirmation such as, "it made coding fun," three said no, five were uncertain, and one didn't respond.

\section{Did Code + Chords software allow your experience with music to be more enjoyable?}

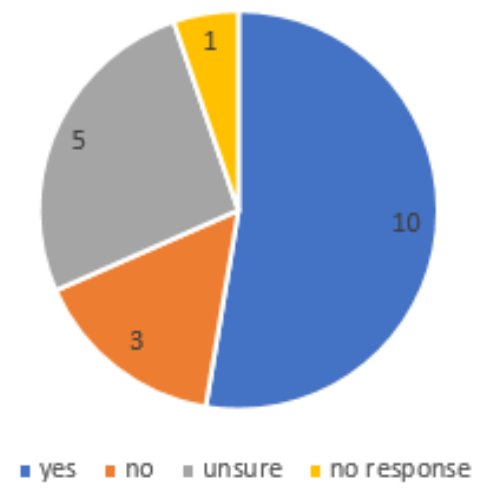

Figure 2: A graph showing post-workshop survey responses to the question, "Did Code + Chords software allow your experience with music to be more enjoyable?" for all participants.

\section{Impact}

Though the results for this study showed an average decrease in self-efficacy, there is a fair amount to take away from the study's outcome. All participants were successfully able to complete each activity throughout the workshop, and self-reflections about their understanding suggest an overall moderate increase in programming knowledge. This shows that each task was achievable for the selected age range, meaning that teaching during the workshop was comprehensive, engagement was sufficient to complete activities, and activities were manageable.

Numerical data from the surveys indicates that the average self-efficacy in participants decreased. This is mainly attributed to large decreases for a few students, such as Banana. For many of the participants, self-efficacy remained static. This shows that the workshops had no effect on self-efficacy for these 
students. 10 out of 19 participants who were asked, "Did Code + Chords software allow your experience with music to be more enjoyable?" responded with a yes or other positive affirmation, whereas only 3 out of 19 said it did not. This shows that although their numerical information did not reflect an increase in self-efficacy, they did communicate having a positive encounter with music technology. Two of the three participants that responded "no" to the question showed up partway through the workshops, which could have contributed to their negative experience with the software.

\section{Improvements}

Reflecting on this study, it is apparent there is ample room for improvement. One of the most important changes the researchers could make to this research would be to target a more specific age-range. This study was designed and approved to welcome ages 7-17 to participate. All participants ended up being 13 or younger, with most being around 9-10 years old. Because the researchers were targeting such a wide age range, the researchers geared activities towards a middle school age group and made activities adaptable for elementary and high school participants. This meant that although the young participants were able to successfully achieve the tasks given to them for each activity, the activities were not necessarily designed to be the most enjoyable for their specific age group. With a narrower target age, the researchers could better customize the activities to most effectively stimulate the students' brains and increase their level of enjoyment while interacting with music technology.

Another critical hindrance to the success of this workshop was the type of data collected. Young participants with minimal experience writing were being asked to write-in answers to seven surveys over the course of two hours, in addition to signing an entry assent form. There was overwhelming documentation of participant experiences and consent, which ended up taking away from the excitement of each activity. In the future, having fewer surveys within such a short time frame would likely keep focus on the experience of each activity instead of on the more mundane documentation of each experience, perhaps increasing the enjoyment of the workshop.

Similarly, the two-hour workshop was far too long to retain participant attention. Researchers found that participants were continually distracted throughout the course of this study. They were the most distracted when they finished activities before their peers, when a researcher was explaining a new concept, or when they were filling out their short surveys. Having a 45-minute workshop that is more concise and focuses on flow would leave less time for distractions and actively encourage participants to engage with new and challenging (yet achievable) ideas.

\section{Acknowledgements}

A special thank you is extended to Playful Learning Lab directors AnnMarie Thomas, Deb Besser, and Jeffrey Jalkio for inspiring this research and guiding the research process. Authors would also like to thank Sarah Muenster-Blakely for working with us to make this study possible. Another thank you is extended to Jenna Laleman, for helping us to create activities to be used in these workshops; Danny Mahota and Emily Meuer, for helping us to develop the code for this workshop; and Grace Kubista, the current Code + Chords project lead. Researchers are grateful to St. Paul Public Libraries for letting us host the workshops in their spaces; ASEE, for allowing us to share our work within their community; and the reader for their continued inquiries into Code + Chords and constant support for the project's improvement. 
References

[1] M. Al Salami, C. Makela, M. Miranda, "Assessing Changes in Teachers' Attitudes toward Interdisciplinary STEM Teaching," International Journal of Technology and Design Education, vol. 27, no. 1, pp. 63-88, 2017. [Online]. Available:

https://www.springerprofessional.de/en/assessing-changes-in-teachers-attitudes-towardinterdisciplinary/7016544 [Accessed February 2nd, 2020].

[2] D. Green, "A Communication Model for the Development of Self-Efficacy in the Classroom," Journal of Teaching in Social Work, vol. 23, no. 3, pp. 107-116, September 2008. [Online]. Available: https://www.tandfonline.com/doi/abs/10.1300/J067v23n03_09 [Accessed February 2nd, 2020].

[3] K. Howland, J. Good, J. Robertson, A. Manches, "Every child a coder?: research challenges for a 5-18 programming curriculum," in Proceedings of the 14th International Conference on Interaction Design and Children, IDC 2015, Medford, MA, USA, June 21-24, 2015, pp. 1-4. 